\title{
COGNITIVE FUNCTION AND FINE MOTOR OUTCOMES IN EXTREMELY PREMATURE US CHILDREN AT AGE 5 YEARS: A LONGITUDINAL STUDY
}

\author{
M. Lee, C. Mcnicholas, J. Pascoe \\ Pediatrics, Wright State University, Dayton, OH, USA
}

Background and aims: The prevalence of extreme prematurity at birth has increased, but little research has examined its impact on developmental outcomes. This study examined the effect of extreme prematurity on kindergarteners' cognitive function and fine motor skills.

Methods: The Early Childhood Longitudinal Study-Birth Cohort, a representative sample of the US children born in 2001 was analyzed for this study. Cognitive function and fine motor skills were compared among 500 extremely premature children (EPC) (gestational age $\leq 30 \mathrm{wks}$ or birthweight $<1000 \mathrm{~g}$ ), 800 premature children (PC), and 5000 term children (TC) $(\geq 37 \mathrm{wks}$ or $\geq 2500 \mathrm{~g})$. Generalized linear regression analyses included sampling weights, children's age, race, sex, socioeconomic status, parental education, and multiple birth status.

Results: Overall mean early reading and math scale scores (SE) were $38.7(0.4)$ and $40.5(0.2)$; $21.6 \%$ failed building a gate and $78.5 \%$ failed all four fine motor tasks. Mean early reading scale scores (MRSS) of EPC was 3.8(0.8) points lower than TC ( $\mathrm{p}$-value $<0.0001$ ) while MRSS of PC was not significantly different from TC. Mean math scores were significantly lower for both EPC $\{4.9(0.6)$, p-value $<0.0001\}$ and PC $\{1.3(0.5)$, p-value $=0.013\}$ compared to TC. EPC were $1.7(95 \% \mathrm{CI}: 1.3-2.2)$ times more likely to fail build a gate and were 2.5(1.7-3.7) times more likely to fail all four tasks compared to TC. Fine motor performance of PC was not different from TC ( $\mathrm{p}$-values $>0.02)$.

Conclusions: Extreme prematurity at birth was associated with cognitive and fine motor delays. This suggests that the biological risk of extreme prematurity persists after adjusting for other factors related to development. 